This is an author-submitted, peer-reviewed version of a manuscript that has been accepted for publication in the European Respiratory Journal, prior to copy-editing, formatting and typesetting. This version of the manuscript may not be duplicated or reproduced without prior permission from the copyright owner, the European Respiratory Society. The publisher is not responsible or liable for any errors or omissions in this version of the manuscript or in any version derived from it by any other parties. The final, copy-edited, published article, which is the version of record, is available without a subscription 18 months after the date of issue publication.

\title{
Minimal clinically important differences in average, best, worst and current intensity and unpleasantness of chronic breathlessness
}

\author{
Magnus Ekström ${ }^{1,2}$ \\ Miriam J Johnson ${ }^{3}$ \\ Chao Huang ${ }^{3}$ \\ David C Currow ${ }^{2,3}$
}

${ }^{1}$ Department of Clinical Sciences, Division of Respiratory Medicine \& Allergology, Lund University, SE-22100 Lund, Sweden

${ }^{2}$ IMPACCT, Faculty of Health, University of Technology Sydney, Ultimo, New South Wales, Australia

${ }^{3}$ Wolfson Palliative Care Research Centre, Hull York Medical School, University of Hull, UK

\section{Corresponding author:}

Magnus Ekström

Department of Medicine, Blekinge Hospital

SE-17385 Karlskrona, Sweden

Telephone: +46455731000

Email pmekstrom@gmail.com

Take Home Message: 
This cohort study determined clinically important differences for current intensity and unpleasantness and the average, best and worst intensity the last 24 hours of chronic breathlessness, which is important for the design of therapeutic trials. 


\begin{abstract}
Background: Chronic breathlessness has devastating consequences. The minimal clinically important difference (MCID) for current intensity has been estimated as $9 \mathrm{~mm}$ on a $100 \mathrm{~mm}$ visual analogue scale (VAS). We aimed to determine MCIDs for commonly used dimensions and recall periods: the current unpleasantness and current, average, best and worst intensity of the last $24 \mathrm{~h}$ for chronic breathlessness.

Methods: This was a secondary analysis of a randomized controlled trial of morphine vs. placebo during seven days in people with chronic breathlessness from severe disease. The breathlessness scores were self-reported using a diary each evening on $100 \mathrm{~mm}$ VAS. The MCID for improvement in each score was estimated using anchor based and distribution based methods.
\end{abstract}

Results: 283 participants (mean age 74.2 years; 63\% males; 58\% COPD; 87.0\% mMRC 3-4) were included. Anchor-based MCIDs for breathlessness scores ranged from $-13.9 \mathrm{~mm}$ to $-9.5 \mathrm{~mm}$. The MCIDs were similar when using different anchors and across all participants, and participants with more severe breathlessness (mMRC 3-4), respectively. Distribution based effect sizes were: small $(-4.7$ to $-6.3 \mathrm{~mm})$, moderate $(-9.4$ to $-12.5 \mathrm{~mm})$ and large effect (-15.0 to $-20.0 \mathrm{~mm})$. Sample sizes for trials using the different scores were proposed. MCIDs of absolute change were more stable than using relative change from baseline.

Conclusion: An improvement of about $10 \mathrm{~mm}$ on a $100 \mathrm{~mm}$ VAS is likely to be clinically meaningful across commonly used measures of chronic breathlessness (current intensity, unpleasantness, and average, best and worst intensity over the last $24 \mathrm{~h}$ ) - to evaluate clinical benefit and effects in therapeutic trials. 


\section{INTRODUCTION}

Chronic breathlessness [1] is a key cause of suffering in advanced disease.[2] Subjective sensations of intensity and unpleasantness may be discerned as distinct aspects by individuals and their impact experienced within the context of duration of the symptom (acute $v s$ chronic) and the meaning attributed by the person. Breathlessness should be measured in routine care as an essential part of disease evaluation to guide best management.[3-5] Although chronic breathlessness cannot be encapsulated in a single quantitative measure,[6] higher scores in uni-dimensional breathlessness scores do predict adverse clinical outcomes and can demonstrate change in response to an intervention, and thus are relevant for patient care and in clinical trials.[7]

Different breathlessness dimensions and recall periods have been used and assessed in trials since a consensus statement on the measurement of breathlessness in advanced disease, [5] including the current intensity (or severity), current unpleasantness, and the average, best or worst intensity during the last 24 hours. [7-15]

The minimal clinically important difference (MCID), defined as the smallest change that is meaningful to the patient, is a key concept for determining the clinical relevance of effects in therapeutic trials.[16] Data are limited on MCIDs for measures of chronic breathlessness.[7] Using an individual pooled data analysis $(n=213)$ of current breathlessness intensity from three trials [17-19] and one dose titration study,[20] Johnson et al. reported a MCID for current intensity of chronic breathlessness of $9 \mathrm{~mm}$ on a $100 \mathrm{~mm}$ visual analogue scale (VAS) using both participant-anchored and distribution-based methods.[7] Distribution-based effect sizes were $5.5 \mathrm{~mm}$ for a small, $11.3 \mathrm{~mm}$ for a moderate, and $18.2 \mathrm{~mm}$ for a large effect.[7] 
The absolute MCID, based on absolute change from baseline, was found to be more stable compared with the relative MCID (which is based on \% change from baseline), and absolute MCIDs were therefore concluded to be preferable for use in trials.[21]

However, we do not know the MCIDs of chronic breathlessness for different frequently used dimensions and temporal periods - the current unpleasantness, and the average, best and worst intensity during the last 24 hours.[22] For example, measures of "current breathlessness" and "usual breathlessness" correlate poorly and likely represent different constructs,[23] and so may have different MCIDs. Further, it is not known whether MCIDs differ by underlying diagnosis (most data pertain to chronic obstructive pulmonary disease [COPD]) as seen in (acute) pain,[24] or in patients with worse activity limitation due to breathlessness, as measured by the modified Medical Research Council (mMRC) scale.[22, 25] This knowledge is of fundamental importance for choosing uni-dimensional assessment scales to reflect patients' experiences in clinical practice and trials.

The primary aim of this paper was to determine MCIDs for improvement in current breathlessness intensity and unpleasantness, and for the average, best and worst intensity during the last 24 hours in participants with advanced disease and chronic breathlessness. Secondary aims were to evaluate MCIDs separately in participants with more severe breathlessness (mMRC 3-4).

\section{METHODS}

\section{Study design and population}


This is a secondary analysis of a randomised, parallel arm, multi-site, fixed dose, placebo controlled, phase III trial of 20mg extended release morphine daily placebo for seven days in patients with chronic breathlessness (Australian New Zealand Clinical Trials Registry, ACTRN12609000806268). The main analysis found no difference in breathlessness between the trial arms when measuring 'breathlessness now'.[14] The database was also previously used to evaluate agreement between breathlessness severity and unpleasantness,[12] compare mMRC ratings between clinicians and participants [26] and to evaluate treatment adverse events.[27]

Participants were recruited from 14 respiratory and palliative care services across Australia in the Australian Government's funded national Palliative Care Clinical Studies Collaborative (PaCCSC).[14] Main eligibility criteria were: age $\geq 18$ years; chronic breathlessness defined as a mMRC [28] breathlessness score $\geq 2$ at screening despite optimal management of underlying cause(s) of breathlessness; stable breathlessness medications for the previous week except 'as needed' medications; an Australia-modified Karnofsky Performance Status (AKPS) scale $\geq 40 ;[29]$ expected survival $\geq 2$ months; and ability to complete a daily diary.[14]

\section{Ethical considerations}

The trial was approved by the Southern Adelaide Clinical Research Ethics Committee (Dnr: EC00188) and the local ethics committee at each site prior to first recruitment at each site. All participants gave their informed written consent to participate and the trial was monitored in accordance with Good Clinical Practice.[30] 


\section{Assessments}

The mMRC breathlessness scale [25] and function using the Australia-modified Karnofsky Performance Scale (AKPS) were rated by the physician at eligibility screening.[26] Other baseline assessments are described elsewhere.[14] Breathlessness scores were rated by participants using $100 \mathrm{~mm}$ horizontal visual analogue scales (VAS) in a diary each evening (current intensity, current unpleasantness, and worst, best, average intensity during the last $24 h)$ at baseline and for the seven days of intervention (low dose morphine or placebo). The VAS is a reliable and valid scale for unidimensional measurement of breathlessness.[31,32] Current intensity was assessed using the question "How is your breathlessness right now?" between 0 ("None") and 100 ("Worst possible"). Current unpleasantness was then assessed using the question "Right now how would you rate the unpleasantness of your breathlessness?" between 0 ("None") and 100 ("The most unpleasant I have ever felt"). The participant was then asked to rate the average, best and worst intensity of breathlessness during the last 24 hours using similar scales as the current intensity.

At the end of the study, participants were asked (double-blinded) whether they had "been less breathless during the past week" ('less breathlessness') and "this medication would benefit me enough to be on it long term" ('benefit') which were used in this current analysis as anchors for determining a participant-defined clinically meaningful change in breathlessness during the study week. The anchors were used line with recommendations to evaluate MCIDs across several anchors and methods [16] and as the questions related to the key concept of a MCID, to measure a change in breathlessness that would be clinically important in terms of patients' experiences and treatment decisions. 


\section{Statistical analyses}

Participant baseline characteristics were summarized by descriptive statistics. For each breathlessness score (current unpleasantness and current, average, best and worst intensity), imputation was performed for those with 1) missing baseline measurements, which was imputed as their first day measurement; 2) missing day seven measurements, which was imputed as the last available value for each score at day five or six.[14] Sensitivity analysis without any imputation was firstly performed. We then imputed the missing values using multiple imputation approach (imputation model set as multivariate normal regression adjusting for baseline characteristic factors; with 20 imputations per case). Both sensitivity analyses yielded similar results.

MCIDs were calculated using anchor-based methods as recommended.[16,33] The 'less breathless' and 'benefit' questions were used as participant anchors of breathlessness change. MCIDs were calculated as the change from baseline to day seven, in the group who affirmed the anchor question (response) compared with the group that did not affirm the question (non-response) for each breathlessness scores with each of the two anchors. The estimates were reported with $95 \%$ confidence intervals (CIs). For each breathlessness score, MCIDs were calculated for all participants and separately for the subgroups with more severe breathlessness (mMRC 3-4).

Distribution-based methods were used to further explore the differences in the scores. The baseline standard deviation (SD) of $0.25,0.5$ and 0.8 were used to define small, medium and large effects.[7] Standard error of measurement (SEM),[32] defined as the baseline SD multiplied by the square root of one minus sample test-retest reliability coefficient, were also 
calculated for comparison purpose. One SEM can be regarded as an estimate of the MCID.[34]

Sample sizes required to detect a MCID change in each breathlessness score with assumed common baseline standard deviation, $90 \%$ power (typically used in definitive trials) and an two-sided alpha $=0.05$ were calculated using nQuery version 8.4 (Statistical Solutions Ltd, Boston, USA). Stata version 15.1 (StataCorp LLC, USA) and R version 3.5 were used for the other statistical analyses.

Relative MCIDs using anchor based methods - defined as a change divided by the score at baseline, were calculated for comparison with the absolute MCIDs (main analysis).[21]

\section{RESULTS}

Of 284 randomised participants, 283 participants (one randomised participant did not meet the inclusion criteria of 'Prognosis of at least 2 months in the opinion of the treating clinician') were included for analysis: mean age 74.2 (SD 9.3) years; 63.2\% males; mean AKPS 61.2 (SD 10.5); main diagnoses were COPD (58.0\%) and cancer (16.6\%); and 87.0\% had a mMRC of 3-4 (Table 1).

Anchor based MCIDs for improvement in the breathlessness scores for the study population ranged from $9.5 \mathrm{~mm}$ to $13.9 \mathrm{~mm}$ (Figure 1). The MCIDs were similar when using the two different participant anchors, as shown by the largely overlapping 95\% CIs (supplemental Table S1). The estimates were also largely similar to the main analysis for sub-group analyses (Supplemental Tables S2-S4). 
Using distribution-based methods, the MCIDs were consistent with small to moderate changes. A small change ranged between $4.7-6.3 \mathrm{~mm}$; moderate change $9.4-12.5 \mathrm{~mm}$; and large change $15.0-20.0 \mathrm{~mm}$ (Table 2). The SEMs for breathlessness ranged from 9.7 to 16.4, slightly higher than the MCIDs calculated by anchor based approach.

Sample sizes required to detect a current MCID's change in each breathlessness score $(90 \%$ power and an alpha $=0.05$ ) are shown in Table 3.

The relative MCIDs ranged from $-26.9 \%$ to $-73.5 \%$ and varied more between the breathlessness scores than the absolute MCIDs (supplemental Table S3).

\section{DISCUSSION}

This study for the first time establishes MCIDs for improvement in a range of commonly used measures of chronic breathlessness - the current intensity and unpleasantness, and the average, best and worst intensity over the last 24 hours. These novel data are important to help interpret symptom response in clinical practice and for valid measurement in interventional trials. Sample size estimations are presented and compared between the measures.

The anchor based MCIDs ranged from $9.5 \mathrm{~mm}$ to $13.9 \mathrm{~mm}$, which is slightly higher than the previous estimate of $9 \mathrm{~mm}(95 \%$ CI 2.1 to 15.8$)$ for current breathlessness.[7] However, the present 95\% CIs overlapped with the earlier estimate.[7] In the present analysis, MCIDs were similar when calculated using two different participant anchors and were consistent with distribution based estimates, which supports the validity of the present findings. Another novel finding is that MCIDs were similar to in the whole population for participants with 
more severe breathlessness (mMRC 3-4). Consistent with the previous analysis by Johnson et al.[21], MCIDs for absolute change were more stable and reliable than the relative MCID (relative change compared to baseline). This study thus confirms that treatment effect should be evaluated using the absolute MCID. Sample sizes (using the present anchor based MCIDs) were calculated for a definite trial (using 90\% power and an alpha of 0.05) and ranged between 61 - 143 participants for different breathlessness scores. Sample sizes were similar in participants with mMRC 3-4. Some previous research suggest that one SEM is equivalent to MCID.[34] In the present analysis, SEMs were consistent with the anchor based estimates, supporting the usage of one SEM to estimate the MCID.

When using a MCID, it should be remembered that while the estimated mean change for the population is most likely to be clinically significant for the individual [16], there is always a degree of uncertainty around the true MCID for the individual. This uncertainty is reflected in the present analysis by the confidence intervals, which were largely overlapping between the compared measures and groups. Taken together, the present findings support an MCID for the different measures of chronic breathlessness around $10 \mathrm{~mm}$ on a $100 \mathrm{~mm}$ VAS (Figure 1).

Strengths of the present analysis include that it is based on the largest RCT of chronic breathlessness to date with self-reported breathlessness scores at similar time points daily over one week. MCIDs were evaluated using two different participant anchors and distribution based methods, as recommended.[16]

Several potential limitations should be noted. It could be argued that the present estimates may not reflect the minimal important differences, as 'minimal' was not included in the anchors. We used available anchors that were considered to relate to a difference that was clinically important ('I have been less breathless during the past week' and 'This medication 
would benefit me enough to be on it long term '). While the true minimal important differences may be slightly smaller, the present estimates are supported by the distribution based analysis that they represent small to moderate effect sizes. Sub groups were too small to evaluate MCIDs in $\mathrm{mMRC} \leq 2$ and in younger patients, which should be investigated in analyses of pooled trial data. As participants were not specifically instructed on the difference between intensity and unpleasantness, the meaning of the upper scale anchors and the ratings could be conflated between intensity and unpleasantness for some participants. The present MCIDs pertain mainly to improvement in breathlessness and cannot be assumed to apply also to deterioration in breathlessness. However, benefit is the most relevant for use in clinics and therapeutic trials.

These findings have several important implications: a change of $10 \mathrm{~mm}$ on a $100 \mathrm{~mm}$ VAS (or 1 unit on a $0-10$ numerical rating scale) is likely to represent a change in chronic breathlessness that is clinically relevant or meaningful for the participant sufficient to inform clinical practice. MCIDs for different breathlessness dimensions and recall periods are given. When designing clinical trials, the use of an absolute MCID is preferred (over relative), and sample sizes are suggested for the different measures of chronic breathlessness. Further research is needed on MCIDs of acute-on-chronic breathlessness, MCIDs for symptom improvement $v s$. worsening and how the MCID is influenced by factors including the baseline severity and history of chronic breathlessness.

\section{ACKNOWLEDGEMENTS}


Funding: ME was supported by an unrestricted grant from the Swedish Society for Medical Research and the Swedish Research Council.

Author contributions: Conception and design: all authors; Data collection: DCC; First draft: ME; Statistical analysis: $\mathrm{CH}$; Interpretation, revision for important intellectual content, and approval of the version to be published: all authors.

Conflicts of interest: DCC is an unpaid member of an advisory board for Helsinn Pharmaceuticals and Specialist Therapuetics, and has consulted to Mayne Pharma and received intellectual property payments from them. MJJ has consulted to Mayne Pharma with institutional payment. ME, $\mathrm{CH}$ and MW have no conflicts of interest to disclose. 


\section{REFERENCES}

1. Johnson MJ, Yorke J, Hansen-Flaschen J, Lansing R, Ekström M, Similowski T, Currow DC. Towards an expert consensus to delineate a clinical syndrome of chronic breathlessness. Eur Respir J 2017; 49(5): pii: 1602277.

2. Moens K, Higginson IJ, Harding R. Are there differences in the prevalence of palliative care-related problems in people living with advanced cancer and eight non-cancer conditions? A systematic review. J Pain Symptom Manage 2014; 48(4): 660-677.

3. Banzett RB, O'Donnell CR. Should we measure dyspnoea in everyone? Eur Respir J 2014; 43(6): 1547.

4. Mularski RA, Campbell ML, Asch SM, Reeve BB, Basch E, Maxwell TL, Hoverman JR, Cuny J, Clauser SB, Snyder C, Seow H, Wu AW, Dy S. A Review of Quality of Care Evaluation for the Palliation of Dyspnea. Am J Respir Crit Care Med 2010; 181(6): 534-538.

5. Dorman S, Jolley C, Abernethy A, Currow D, Johnson M, Farquhar M, Griffiths G, Peel T, Moosavi S, Byrne A, Wilcock A, Alloway L, Bausewein C, Higginson I, Booth S. Researching breathlessness in palliative care: consensus statement of the National Cancer Research Institute Palliative Care Breathlessness Subgroup. Palliat Med 2009; 23(3): 213-227.

6. Carel H. Breathlessness: the rift between objective measurement and subjective experience. The Lancet Respir Med 2018; 6(5): 332-333.

7. Johnson MJ, Bland JM, Oxberry SG, Abernethy AP, Currow DC. Clinically important differences in the intensity of chronic refractory breathlessness. J Pain Symptom Manage 2013; 46(6): 957-963.

8. Boucly A, Morélot-Panzini C, Garcia G, Weatherald J, Jaïs X, Savale L, Montani D, Humbert M, Similowski T, Sitbon O, Laveneziana P. Intensity and quality of exertional dyspnoea in patients with stable pulmonary hypertension. Eur Respir J. 2019 Nov 26. pii: 1802108. doi: 10.1183/13993003.02108-2018.

9. Morélot-Panzini C, Gilet H, Aguilaniu B, Devillier P, Didier A, Perez T, Pignier C, Arnould B, Similowski T. Real-life assessment of the multidimensional nature of dyspnoea in COPD outpatients. Eur Respir J. 2016; 47(6): 1668-1679.

10. Seow H, Barbera L, Sutradhar R, Howell D, Dudgeon D, Atzema C, Liu Y, Husain A, Sussman J, Earle C. Trajectory of performance status and symptom scores for patients with cancer during the last six months of life. J Clin Oncol. 2011; 29(9): 1151-1158.

11. Currow DC, Ekstrom M, Louw S, Hill J, Fazekas B, Clark K, Davidson PM, McDonald C, Sajkov D, McCaffrey N, Doogue M, Abernethy AP, Agar M. Sertraline in symptomatic chronic breathlessness: a double blind, randomised trial. Eur Respir J 2019: 53(1); pii 1801270. 
12. Ekström M, Williams M, Johnson MJ, Huang C, Currow DC. Agreement Between Breathlessness Severity and Unpleasantness in People With Chronic Breathlessness: A Longitudinal Clinical Study. J Pain Symptom Manage 2019; 57(4): 715-723.e5.

13. Johnson MJ, Cockayne S, Currow DC, Bell K, Hicks K, Fairhurst C, Gabe R, Torgerson D, Jefferson L, Oxberry S, Ghosh J, Hogg KJ, Murphy J, Allgar V, Cleland JGF, Clark AL. Oral modified release morphine for breathlessness in chronic heart failure: a randomized placebo-controlled trial. ESC Heart Fail 2019 Aug 6. doi: 10/1002/ehf2. 12498.

14. Currow D, Louw S, McCloud P, Fazekas B, Plummer J, McDonald CF, Agar M, Clark K, McCaffery N, Ekstrom MP. Regular, sustained-release morphine for chronic breathlessness: a multicentre, double-blind, randomised, placebo-controlled trial. Thorax 2020; 75: 50-56.

15. Abernethy AP, McDonald CF, Frith PA, Clark K, Herndon JE, 2nd, Marcello J, Young IH, Bull J, Wilcock A, Booth S, Wheeler JL, Tulsky JA, Crockett AJ, Currow DC. Effect of palliative oxygen versus room air in relief of breathlessness in patients with refractory dyspnoea: a double-blind, randomised controlled trial. Lancet 2010; 376(9743): 784-793.

16. Guyatt GH, Osoba D, Wu AW, Wyrwich KW, Norman GR. Methods to explain the clinical significance of health status measures. Mayo Clin Proc 2002; 77(4): 371-383.

17. Abernethy AP, Currow DC, Frith P, Fazekas BS, McHugh A, Bui C. Randomised, double blind, placebo controlled crossover trial of sustained release morphine for the management of refractory dyspnoea. BMJ 2003; 327(7414): 523-528.

18. Currow DC, McDonald C, Oaten S, Kenny B, Allcroft P, Frith P, Briffa M, Johnson MJ, Abernethy AP. Once-Daily Opioids for Chronic Dyspnea: A Dose Increment and Pharmacovigilance Study. J Pain Symptom Manage 2011; 42(3): 388-399.

19. Johnson MJ, McDonagh TA, Harkness A, McKay SE, Dargie HJ. Morphine for the relief of breathlessness in patients with chronic heart failure--a pilot study. Eur J Heart Fail 2002; 4(6): 753-756.

20. Oxberry SG, Torgerson DJ, Bland JM, Clark AL, Cleland JG, Johnson MJ. Short-term opioids for breathlessness in stable chronic heart failure: a randomized controlled trial. Eur J Heart Fail 2011; 13(9): 1006-1012.

21. Johnson MJ, Bland JM, Oxberry SG, Abernethy AP, Currow DC. Measuring improvement in dyspnoea: should absolute or relative values be used? Eur Respir J 2014; 44: $1700-1703$.

22. Wade J, Mendonca S, Booth S, Ewing G, Gardener AC, Farquhar M. Are within-person Numerical Rating Scale (NRS) ratings of breathlessness 'on average' valid in advanced disease for patients and for patients' informal carers? BMJ Open Respir Res 2017; 4(1): e000235. 
23. Gift AG, Narsavage G. Validity of the numeric rating scale as a measure of dyspnea. Am J Crit Care 1998; 7(3): 200-204.

24. Olsen MF, Bjerre E, Hansen MD, Hilden J, Landler NE, Tendal B, Hrobjartsson A. Pain relief that matters to patients: systematic review of empirical studies assessing the minimum clinically important difference in acute pain. BMC Med 2017; 15(1): 35.

25. Bestall JC, Paul EA, Garrod R, Garnham R, Jones PW, Wedzicha JA. Usefulness of the Medical Research Council (MRC) dyspnoea scale as a measure of disability in patients with chronic obstructive pulmonary disease. Thorax 1999; 54(7): 581-586.

26. Ekström M, Chang S, Johnson MJ, Fazekas B, Kochovska S, Huang C, Currow DC. Low agreement between mMRC rated by patients and clinicians - implications for practice. Eur Respir J 2019; 55(1): 10.1183/13993003.01517-2019.

27. Johnson MJ, Sbizzera I, Fairhurst C, Fazekas B, Agar M, Ekström M, Currow DC. No excess harms from sustained release morphine: a randomised placebo-controlled trial in chronic breathlessness. BMJ Supportive \& Palliative Care 2019. doi:

10.1136/bmjspcare-2019-002009.

28. Brooks SM. Surveillance for respiratory hazards. ATS News 1982; 8: 12-16.

29. Abernethy A, Shelby-James T, Fazekas B, Woods D, Currow D. The Australia-modified Karnofsky Performance Status (AKPS) scale: a revised scale for contemporary palliative care clinical practice [ISRCTN81117481]. BMC Palliat Care 2005; 4(1): 7 .

30. European Medicines Agency. ICH Harmonised Tripartite Guideline E6: Note for Guidance on Good Clinical Practice (PMP/ICH/135/95) London: European Medicines Agency; 2002.

31. Gift AG. Validation of a vertical visual analogue scale as a measure of clinical dyspnea. Rehabil Nurs 1989; 14(6): 323-325.

32. Parshall MB, Schwartzstein RM, Adams L, Banzett RB, Manning HL, Bourbeau J, Calverley PM, Gift AG, Harver A, Lareau SC, Mahler DA, Meek PM, O'Donnell DE, American Thoracic Society Committee on D. An official American Thoracic Society statement: update on the mechanisms, assessment, and management of dyspnea. Am J Respir Crit Care Med 2012; 185(4): 435-452.

33. Crosby RD, Kolotkin RL, Williams GR. Defining clinically meaningful change in health-related quality of life. J Clin Epidemiol 2003; 56(5): 395-407.

34. de Vet HC, Terwee CB, Ostelo RW, Beckerman H, Knol DL, Bouter LM. Minimal changes in health status questionnaires: distinction between minimally detectable change and minimally important change. Health and quality of life outcomes 2006; 4: 54. 
Table 1. Baseline characteristics

\begin{tabular}{|c|c|c|}
\hline Characteristic & & $\mathrm{N}=\mathbf{2 8 3}$ \\
\hline \multirow[t]{2}{*}{ Age } & Mean (SD) & $74.2(9.3)$ \\
\hline & Min, $\max$ & $44.3,94.1$ \\
\hline Gender, n (\%) & Female & $104(36.8 \%)$ \\
\hline \multirow{2}{*}{$\begin{array}{l}\text { Performance status } \\
\text { (AKPS) }\end{array}$} & Mean (SD) & $61.2(10.5)$ \\
\hline & Min, $\max$ & 3,90 \\
\hline \multirow{2}{*}{ BMI $\left(\mathrm{kg} / \mathrm{m}^{2}\right)$} & Mean (SD) & $25.5(7.3)$ \\
\hline & Min, $\max$ & $12.3,66.1$ \\
\hline \multirow{4}{*}{$\begin{array}{l}\text { mMRC } \\
\text { breathlessness } \\
\text { score at baseline } \\
\text { rated by clinician, } n \\
(\%)\end{array}$} & 2 & $22(7.8 \%)$ \\
\hline & 3 & $140(49.5 \%)$ \\
\hline & 4 & $106(37.5 \%)$ \\
\hline & Missing & $15(5.3 \%)$ \\
\hline \multirow{5}{*}{$\begin{array}{l}\text { Primary cause of } \\
\text { breathlessness, } \\
\mathrm{n}(\%)\end{array}$} & COPD & $164(58.0 \%)$ \\
\hline & Cancer & $47(16.6 \%)$ \\
\hline & Cardiac failure & $4(1.4 \%)$ \\
\hline & Mixed & $37(13.1 \%)$ \\
\hline & Other & $31(11.0 \%)$ \\
\hline \multirow{2}{*}{$\begin{array}{l}\text { Charlson } \\
\text { Co-morbidity } \\
\text { Index }\end{array}$} & Mean (SD) & $3.3(2.5)$ \\
\hline & Min, $\max$ & 0,13 \\
\hline Oxygen use & Yes n (\%) & $161(56.9 \%)$ \\
\hline $\begin{array}{l}\text { Smoking status, } \\
\mathrm{n}(\%)\end{array}$ & Never smoked & $49(17.3 \%)$ \\
\hline
\end{tabular}




\begin{tabular}{|l|l|c|}
\hline & Ex-smoker & $199(70.3 \%)$ \\
\cline { 2 - 3 } & Current smoker & $33(11.7 \%)$ \\
\cline { 2 - 3 } & Missing & $2(0.7 \%)$ \\
\hline
\end{tabular}

Abbreviations: AKPS, Australia modified Karnofsky performance scale; BMI, body mass index; mMRC, modified Medical Research Council scale; SD, standard deviation. 
Table 2. Distribution-based analysis of clinically important differences in different breathlessness measures

\begin{tabular}{lc:cccc}
\hline Dimension & Unpleasantness & \multicolumn{4}{c}{ Intensity } \\
\hline Focal period & Current & Current & Average & Best & Worst \\
\hline Small change (0.25 SD baseline) & 6.1 & 6.3 & 4.7 & 5.4 & 6.0 \\
$\begin{array}{l}\text { Moderate change (0.5 SD } \\
\text { baseline) }\end{array}$ & 12.2 & 12.5 & 9.4 & 10.8 & 12.0 \\
Large change (0.8 SD baseline) & 19.5 & 20.0 & 15.0 & 17.3 & 19.1 \\
\hdashline SEM & 14.6 & 16.4 & 9.7 & 12.2 & 14.9 \\
& & & & & \\
\hline
\end{tabular}

Estimates are $\mathrm{mm}$ on a $100 \mathrm{~mm}$ visual analogue scale (VAS).

Abbreviations: SD, standard deviation; SEM, standard error of measurement; 
Table 3. Sample sizes for breathlessness scores using participant-anchored MCIDs for each breathlessness score as mean difference and common baseline standard deviation as SD, at 5\% two-sided significant level and $90 \%$ power

\begin{tabular}{|c|c|c|c|c|c|c|c|}
\hline \multirow{2}{*}{$\begin{array}{l}\text { Dimension } \\
\text { Focal period }\end{array}$} & & & \multirow{2}{*}{$\begin{array}{c}\text { Unpleasantness } \\
\text { Current }\end{array}$} & \multicolumn{4}{|c|}{ Intensity } \\
\hline & & & & Current & Average & Best & Worst \\
\hline $\begin{array}{l}\text { Participant } \\
\text { preference } \\
\text { question }\end{array}$ & Group & Parameter & & & & & \\
\hline \multirow{6}{*}{$\begin{array}{l}\text { 'I have been } \\
\text { less breathless } \\
\text { during the past } \\
\text { week' }\end{array}$} & \multirow{3}{*}{ All } & $M C I D$ & -10.9 & -13.9 & -9.5 & -12.4 & -10.9 \\
\hline & & Baseline SD & 25.1 & 24.4 & 19.8 & 21.3 & 24.3 \\
\hline & & $\begin{array}{l}\text { Sample } \\
\text { size }\end{array}$ & 113 & 66 & 92 & 63 & 106 \\
\hline & \multirow{3}{*}{$\begin{array}{l}\mathrm{mMRC} \\
3,4\end{array}$} & $M C I D$ & -10.7 & -14.5 & -8.7 & -12.7 & -11.1 \\
\hline & & Baseline SD & 24.5 & 24.5 & 19.7 & 21.2 & 24.4 \\
\hline & & $\begin{array}{l}\text { Sample } \\
\text { size }\end{array}$ & 112 & 61 & 109 & 60 & 103 \\
\hline \multirow{6}{*}{$\begin{array}{l}\text { 'This } \\
\text { medication } \\
\text { would benefit } \\
\text { me enough to } \\
\text { be on it long } \\
\text { term' }\end{array}$} & \multirow{3}{*}{ All } & $M C I D$ & -10.2 & -9.9 & -11.4 & -12.2 & -11.3 \\
\hline & & Baseline SD & 26.5 & 24.4 & 19.7 & 21.2 & 24.3 \\
\hline & & $\begin{array}{l}\text { Sample } \\
\text { size }\end{array}$ & 143 & 129 & 64 & 65 & 99 \\
\hline & & $M C I D$ & -10.1 & -9.5 & -10.9 & -13.0 & -11.4 \\
\hline & $\mathrm{mMRC}$ & Baseline SD & 24.5 & 24.4 & 19.7 & 21.2 & 24.4 \\
\hline & 3,4 & $\begin{array}{l}\text { Sample } \\
\text { size }\end{array}$ & 125 & 140 & 70 & 57 & 98 \\
\hline
\end{tabular}

Abbreviations: MCID, minimal clinically important difference; mMRC, modified Medical Research Council scale 
